\title{
The physics of Magnus gliders
}

\author{
Nicolas Plihon, ${ }^{*}$ Gauthier Legrand, Francis Pagaud, Arsène Chemin, Jérémy Ferrand, and Nicolas Taberlet \\ Univ Lyon, ENS de Lyon, Univ Claude Bernard Lyon 1, \\ CNRS, Laboratoire de Physique, F-69342 Lyon, France
}

(Dated: August 26, 2021)

\begin{abstract}
Magnus gliders are spinning toys displaying spectacular looped trajectories when launched at large velocity. These trajectories originate from the large amplitude of the Magnus force due to translational velocities of a few meters per second combined with backspin of a few hundred radians per seconds. In this article we analyse the trajectories of Magnus gliders built from paper cups, easily reproducible in the laboratory. We highlight an analogy between the trajectory of the glider and the trajectory of charged particles in crossed electric and magnetic fields. The influence of the initial velocity and the initial backspin on the trajectories is analyzed using high speed imaging. The features of these trajectories are captured by a simple model of the evolution of the Magnus and drag forces as a function of the spin of the gliders. The experimental data and the modeling show that the type of trajectories - for instance the occurence of loops - depends mostly on the value and orientation of the initial translational velocity, regardless of the value of the backspin, while the maximum height of the apex depends on both the initial translational velocity and initial backspin.
\end{abstract}

\section{INTRODUCTION}

Spinning objects moving rapidly in air often display complex trajectories due to the non-linear evolution of the aerodynamic forces they experience. In addition to the drag (present regardless of the spin rate), the rotation induces a force that is perpendicular to the velocity, known as the Magnus effect. The first explanation of the Magnus effect is often attributed to German physicist Heinrich Gustav Magnus [1], although a number of scientists (including Sir Isaac Newton) described the phenomenon far earlier [2].

The combined effect of spin and drag on translating objects is extraordinarily important in sports [3, 4], and has attracted much attention in the context of soccer $[5,6]$, baseball [7], golf [8, 9], tennis [10], but also badminton and the dynamics of shuttlecocks $[11,12]$ or other games involving spinning objects without spherical symmetry, exemplified by frisbees or boomerangs [13]. Indeed, the trajectory of lifted balls are sometimes puzzling for sport players, such as free kicks in soccer [14], paradoxical pop-ups in baseball [15] or zigzagging paths at very low spin [16]. In this context, the dimensionless number controlling the amplitude of the Magnus force is referred to as the spin number $S_{p}$ [17].

In this article, we analyze and model the trajectories of a spinning toy, known as the Magnus glider (or Magnus flyer), widely disseminated thanks to a popular video by Bruce Yeany [18] (see also supplementary video). A Magnus glider typically consists of two light cups with their bottoms glued together, launched (using a rubber band) at a high velocity (typically $10 \mathrm{~m} / \mathrm{s}$ ) with high backspin (typically $100 \mathrm{rev} / \mathrm{s}$ ). Due to its initial backspin, the flyer experiences a Magnus force that remains perpendicular to the velocity, sometimes resulting in looped trajecto-

* nicolas.plihon@ens-lyon.fr

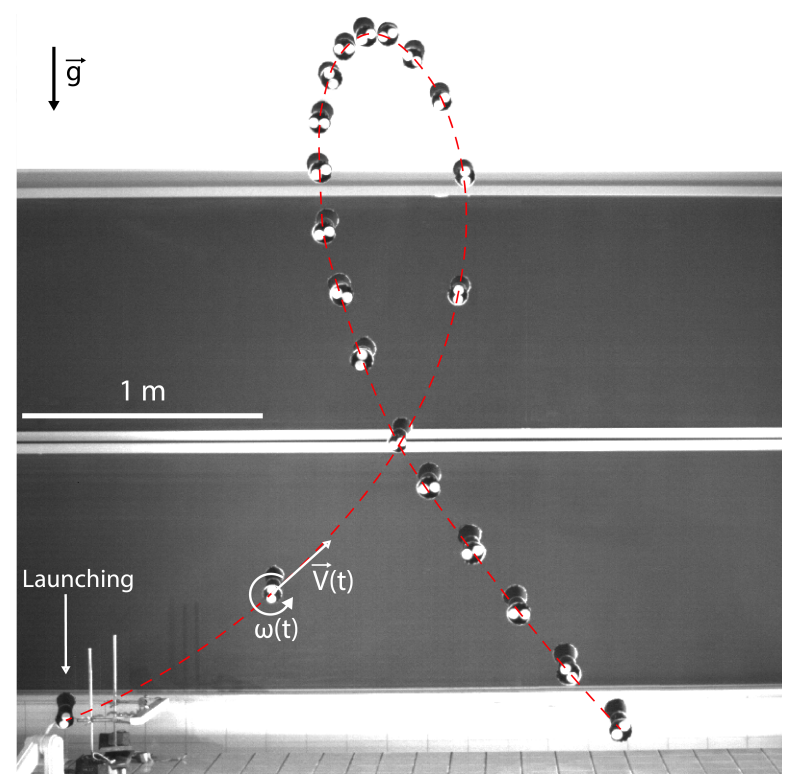

FIG. 1. Chrono-photography showing the positions of the Magnus glider every $75 \mathrm{~ms}$, after launching from the bottom left corner. The trajectory (dashed red line) displays a loop.

ries. It should be emphasized that, contrary to conventional gliders, the Magnus flyer only experiences lift due to its initial rotational kinetic energy which eventually dissipates -Magnus glider is therefore a misnomer, but the term is retained here due to its popular use in spite of its inadequacy. Further technical details of the Magnus glider itself and launching procedure are given in section II and in the supplementary video. Fig. 1 shows chronophotography of the successive positions of the Magnus glider (every $75 \mathrm{~ms}$ ), initially launched at a velocity of $15 \mathrm{~m} / \mathrm{s}$ and a spin rate of $660 \mathrm{rad} / \mathrm{s}$ : the backspin generates a Magnus lift force strong enough to lead to this spectacular looped trajectory. Magnus gliders are thus a unique tool to investigate the various complex trajec- 
tories observed for sports-balls at the laboratory scale, with two major advantages: the first one being that trajectories remain two dimensional, since a Magnus glider is much closer to a cylinder than to a ball; this is a strong asset for imaging trajectories. In this paper, we will only work in the vertical 2D-plane and any lateral motion is ignored. This is in general a very good assumption since experimentally all flights remains planar, but any asymmetry in the flyer itself may lead to lateral instability. The second advantage is that complex trajectories may be easily imaged since the typical spatial scale is of the order of a few meters (as compared to tens of meters or more for usual sports-balls trajectories) and launching Magnus gliders does not require advanced technical skills (as compared, for instance, to the long practice required to pitch baseballs).

The importance of the Magnus effect is not restricted to sports-balls trajectories, and several important studies were led in the context of aeronautical or marine engineering applications such as Flettner boats [19] or airplanes [20]. Here, we introduce a simple educational tool allowing for the investigation of the Magnus force and its features, accessible at the laboratory scale.

The article is organized as follows. The details of the experimental protocol and image analysis are provided in section II. In section III, the trajectories of several experimental launches are computed using a model of the aerodynamic forces using data compiled from previous aerodynamical studies. Other features of the trajectories, such as oscillations and the spin-down of the glider due to drag, are then analyzed. Finally, the conditions for obtaining looped trajectories similar to the one shown in Fig. 1 as a function of the various control parameters are discussed in section IV. Section V concludes the article.

\section{EXPERIMENTAL TRAJECTORIES OF MAGNUS GLIDERS}

A sketch of the Magnus glider used in our study is provided in Fig. 2(a). It is made from two paper cups of height $L / 2=9 \mathrm{~cm}$, bottom radius $2.75 \mathrm{~cm}$, and top radius $4 \mathrm{~cm}$, glued together at their bottoms. The glider has an average radius $R=3.37 \mathrm{~cm}$ and a total length $L=18.8 \mathrm{~cm}$. In order to ease the detection of trajectories and angular velocities from high speed video-imaging, the Magnus glider is painted black and paper end-caps with two large white disks are glued to its sides (see Fig. 1 and the supplementary video for further details, as well as the appendix A). The total mass of the Magnus glider is $m=36.8 \mathrm{~g}$. The initial spin and propulsion are provided using a 1-m-long rubber band wrapped around the center of the Magnus glider (the bottom of each cup), attached to a fixed point and stretched to launch the assembly, as sketched in Fig. 2(b) (see supplementary video). The rubber band is wrapped around the glider with constant tension provided by a weight attached at the bottom the band (see the supplementary video and appendix A for

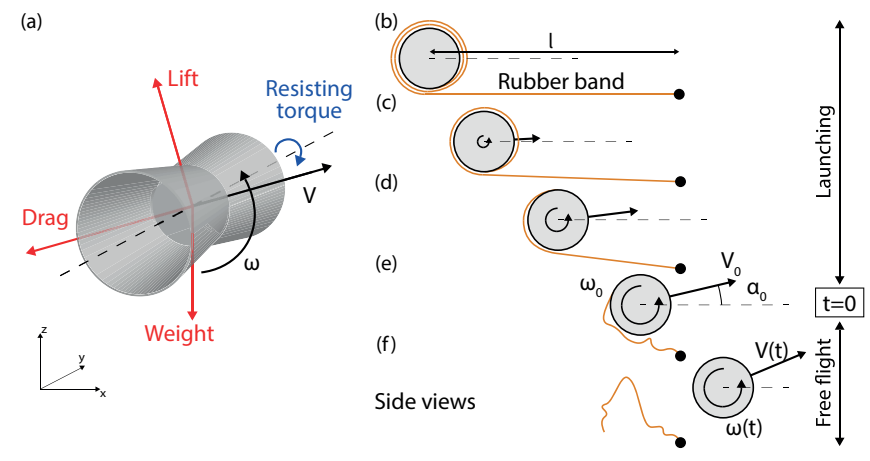

FIG. 2. (a) Sketch of the Magnus glider showing the forces acting during free flight. (b)-(f) Time sequence showing the launching protocol using a rubber band. The black point represents a fixed point.

details). The Magnus glider is then held at a distance $l$ from the fixed point, providing an initial elastic tension to the remaining part of the rubber band which will create forward thrust. As the rubber band contracts and unwinds from the glider, the glider gradually acquires back-spin and linear velocity, as sketched in Fig. 2(c)-(f). For each run, the reference time $t=0$ is defined as the time at which the rubber band is completely unwound and has no contact with the glider. At time $t=0$, the glider has an initial velocity $\mathbf{V}_{\mathbf{0}}$ and initial angular velocity $\omega_{0}$. The initial velocity $\mathbf{V}_{\mathbf{0}}$ has an amplitude $V_{0}$, and makes an angle $\alpha_{0}$ with the horizontal (Fig. 2(e)). The values of $\mathbf{V}_{\mathbf{0}}$ and $\omega_{0}$ depend on three parameters: (1) the number of turns of the rubber band around the Magnus glider, (2) the mass of the weight attached to the bottom of the band, (3) the tension of the remaining part of the rubber band. Note that during the launch phase (steps (c)-(f) in Fig. 2), a (Magnus) lift force is exerted on the Magnus glider. Its origin is that a flow is driven by the rotation of the glider: outside of the boundary layer, and in the center of mass reference frame, the flow velocity at the top of the glider is larger than the flow velocity at the bottom of the glider due to the addition of the translational speed to the flow driven by rotation. Due to the difference in flow velocity, a pressure gradient exerts a vertical force directed upwards. As a consequence, the initial launch angle is not directly set by the angle at which the rubber band is stretched since the glider experiences a lift force during the launch procedure $(t<0)$. The values of $V_{0}, \alpha_{0}$ and $\omega_{0}$ are therefore computed $a$ posteriori from image processing of the videos.

The trajectories are recorded using a Phantom v2511 high speed camera, at a rate of $4000 \mathrm{fps}$, with a resolution of 1280 by 800 pixels. We stress here that the trajectories of the Magnus gliders could be imaged with cameras available on mid-range smartphones as discussed in appendix B. Each image is processed to extract the locations of the centers $\mathbf{r}_{\mathbf{1}}(t)$ and $\mathbf{r}_{\mathbf{2}}(t)$ of the two white disks on the end-plate [21]. For each image, the location of the center $\mathbf{r}(t)$ of the Magnus glider is determined as 
the average $\left(\mathbf{r}_{\mathbf{1}}(t)+\mathbf{r}_{\mathbf{2}}(t)\right) / 2$, while the angular position $\theta(t)$ of the Magnus glider is determined as the angle between $\mathbf{r}_{\mathbf{2}}(t)-\mathbf{r}_{\mathbf{1}}(t)$ and the horizontal (see supplementary videos for details). The location of the Magnus glider at time $t$ is noted $\mathbf{r}(t)=x(t) \hat{\mathbf{x}}+z(t) \hat{\mathbf{z}}$, where $\hat{\mathbf{x}}$ is the horizontal direction and $\hat{\mathbf{z}}$ is the upward vertical direction. The instantaneous velocity $\mathbf{V}(t)$ and the instantaneous angular velocity $\omega(t)$ are computed from $\mathbf{r}(t)$ and $\theta(t)$.

For the regimes reported in this article $V_{0}$ is of the order of $10 \mathrm{~m} / \mathrm{s}$, while the typical angular velocity $\omega_{0}$ is of the order of $500 \mathrm{rad} / \mathrm{s}$. Introducing $\nu$ as the kinematic viscosity of air, the features of the flow created by the Magnus glider, and its trajectory, depend upon two dimensionless numbers, namely the Reynolds numbers $R e$ defined as

$$
R e=\frac{2 R V}{\nu}
$$

and the Spin number $S_{p}$ defined as

$$
S_{p}=\frac{\omega R}{V} .
$$

The Reynolds number at time $t \simeq 0$ is in excess of $5 \times$ $10^{4}$, which means that the flow is fully turbulent. For the experiments reported in this study, the Spin number is always larger than 2. From previous studies in wind tunnels [22, 23], we know that the values of the drag and lift forces depend on the geometrical parameters and on the values of $S_{p}$ and Re.

Figure 3 shows the three types of trajectories of the Magnus glider obtained for increasing values of the initial velocity $\mathbf{V}_{\mathbf{0}}$ and angular velocity $\omega_{0}$ from top to bottom (see caption). The trajectory in the top panel (lowest speed and spin rate) has the shape of an arch and will be referred to as an arch, that in the central panel as a cusp and that in lower panel as a loop.

The time evolution of the angular velocity $\omega$ of the Magnus glider is reported in the second column of Fig. 3. The red dots indicate $\omega=d \theta(t) / d t$, computed using a Savitsky-Golay of first order on 81 points (which corresponds to a low-pass filter with $50 \mathrm{~Hz}$ cut-off frequency) and the black dashed line is an empirical fit of the form $\omega_{0} \exp \left(-t / \tau_{0}\right)$. Although the decrease in rotation speed is very clear, the fractional decrease is small over the duration of a typical experiment as $\tau_{0}$ is of the order of a few seconds. In the following, the change in angular velocity is taken into account but a simpler assumption of constant rotation speed yields very satifactory results as well (see next section for a thorough discussion). The rightmost column displays the velocity $V(t)$ (solid red lines), computed using a Savitsky-Golay of first order on 401 points (which corresponds to a low-pass filter with a 10 $\mathrm{Hz}$ cut-off frequency) as well as the time evolution of the Spin number $S_{p}$ (dotted black line), using the functional fit $\omega_{0} \exp \left(-t / \tau_{0}\right)$ for the angular velocity. The velocity initially decreases due to drag and since the Magnus glider flies upward, part of its initial kinetic energy is converted into potential energy. Clearly, a strong energy dissipation is caused by the drag forces: the velocity at the end of the trajectories, at $z=0$ (i.e. at time $t \simeq 1.3$ s) typically only amounts to one third of the the initial velocity, also at $z=0$. We note that the Spin number is always greater than 2 , and its initial value quickly increases since the velocity decreases. We also note that, for the cusp regime, the velocity vanishes at the apex, and as a consequence the Spin number diverges. A first observation is that regimes displaying a cusp or a loop require high initial linear and angular velocities; the exact conditions will be elucidated in section IV.

\section{MODELING THE AERODYNAMIC FORCES ACTING ON MAGNUS GLIDERS}

\section{A. Predicting trajectories from drag and lift forces}

In this section, we discuss the features of the aerodynamic forces required to adequately model the trajectory of the Magnus glider. The equations of motion applied to the center of mass of the Magnus glider $\mathbf{r}(t)$ are:

$$
m \mathbf{a}=m \mathbf{g}+\mathbf{F}_{L}+\mathbf{F}_{D},
$$

where $\mathbf{a}$ is the Magnus glider acceleration, $\mathbf{F}_{L}$ is the lift force due to the Magnus effect and $\mathbf{F}_{D}$ the drag force.

Before discussing realistic models, it is instructive to address the simplest model of an ideal fluid of density $\rho$, for which the flow features around the Magnus glider may be described using potential flow theory [24]. In the (non-inertial) center of mass reference frame, the flow is expressed as the addition of a vortex flow surrounding the glider with an uniform incoming flow (due to the glider translation). Since there is no viscosity, there is no drag exerted on the Magnus glider, and, restricting the computation to a two-dimensional case, the lift force due to Magnus effect reads $\mathbf{F}_{L}=\rho \mathbf{V}(t) \times \boldsymbol{\Gamma}$, where $\boldsymbol{\Gamma}$ is the circulation of the flow along the cylinder, defined as $\boldsymbol{\Gamma}=2 \pi \boldsymbol{\omega}_{0} R^{2} L$. Therefore, in the framework of potential flow theory, the Magnus force is proportional to the product of the translational velocity with the rotational velocity. In the presence of forces perpendicular to the velocity, trajectories obtained from integration of the equations of motion are known as trochoids (a generic family of curves including the cycloid). These trajectories are well known in the context of plasma physics for the guiding center dynamics of single charged particles in the presence of a constant magnetic field $\mathbf{B}_{0}[25,26]$. Indeed, in the presence of a constant electric field $\mathbf{E}_{0}$ perpendicular to $\mathbf{B}_{0}$, the equations of motion for a particule of mass $m_{q}$ and charge $q$ reads $m_{q} \mathbf{a}=q \mathbf{E}_{0}+q \mathbf{V} \times \mathbf{B}_{\mathbf{0}}$, which is analogous to that of the Magnus glider in the framework of an ideal fluid (the electric force $q \mathbf{E}_{0}$ being analogous to gravitational force $m \mathbf{g}$ and the Lorentz force $q \mathbf{V} \times \mathbf{B}_{\mathbf{0}}$ being analogous to the Magnus force $\left.\rho \mathbf{V}(t) \times \boldsymbol{\Gamma}\right)$. 

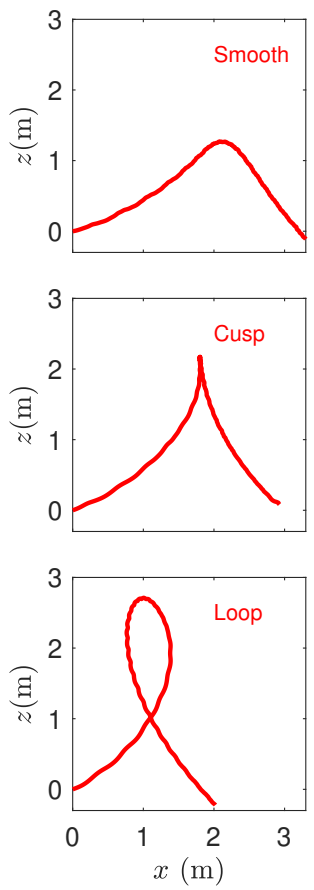
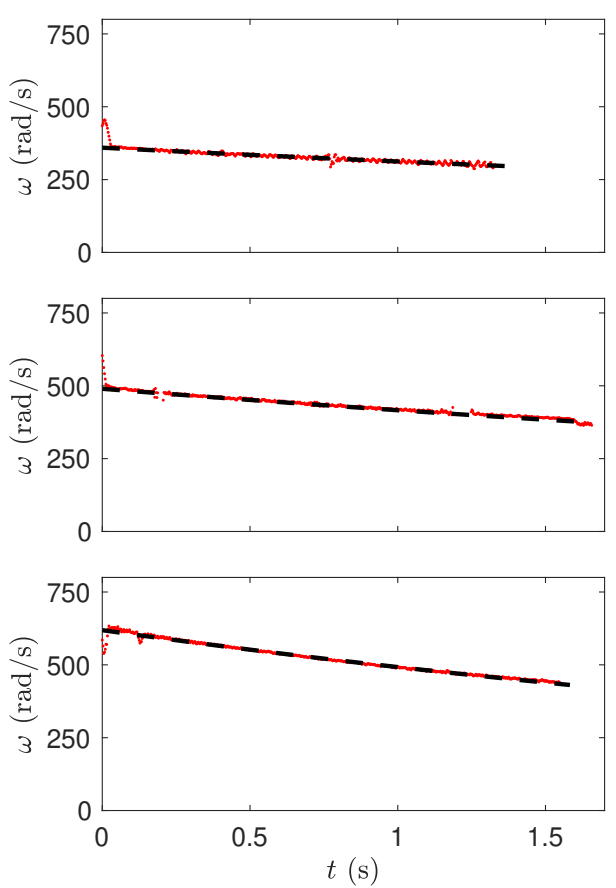
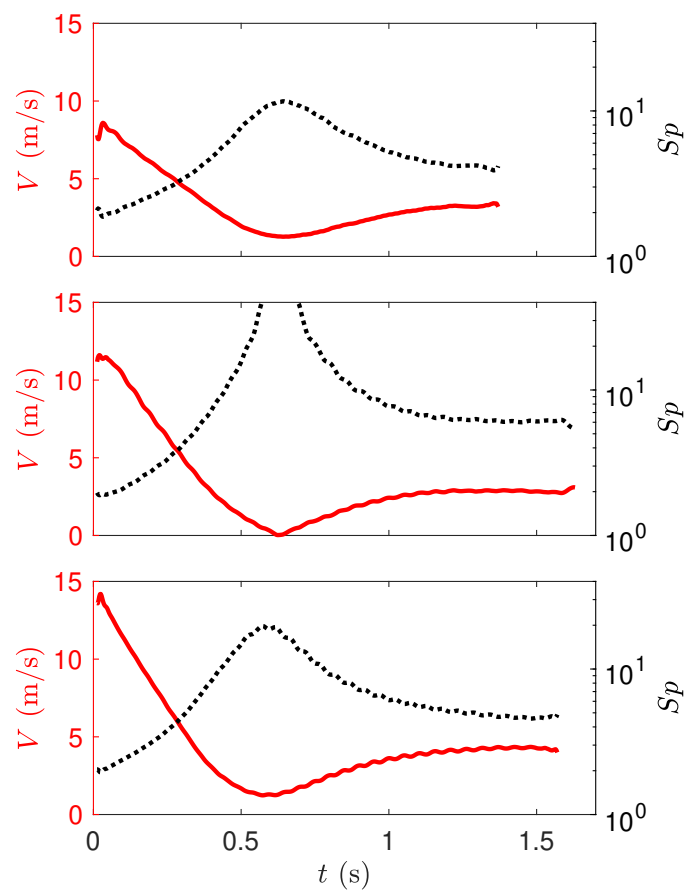

FIG. 3. (left) Trajectory, (center) time evolution of the angular velocity $\omega$, and (right) time evolution of the amplitude of the velocity $V(t)$ (solid red line) and spin number $S_{p}(t)$ (dotted black line) for three launches of the Magnus glider. The measured initial conditions are (top) $\omega_{0}=362 \mathrm{rad} / \mathrm{s}, V_{0}=8.4 \mathrm{~m} / \mathrm{s}$ and $\alpha_{0}=18^{\circ}$, (middle) $\omega_{0}=492 \mathrm{rad} / \mathrm{s}, V_{0}=11.9 \mathrm{~m} / \mathrm{s}$ and $\alpha_{0}=21^{\circ}$ and (bottom) $\omega_{0}=623 \mathrm{rad} / \mathrm{s}, V_{0}=15 \mathrm{~m} / \mathrm{s}$ and $\alpha_{0}=29^{\circ}$.

The charged particle gyrates at the cyclotron frequency around a guiding center drifting perpendicularly to the electric field and the magnetic field - an effect known as the electric drift in plasma physics. The radius of gyration is known as the Larmor radius, and is by the amplitude of the velocity perpendicular to the magnetic magnetic. Applying this analogy to the context of Magnus gliders leads to a drift perpendicular to the gravitational field and spin axis. This simple model of inviscid flow has the pedagogical virtue of being analytically solvable, with trajectories symmetric around the apex. As already pointed out, due to the existence of drag, the experimental trajectories reported here are not symmetric around the apex and a further degree of refinement is required to model the dynamics of the Magnus glider.

A more detailed model incorporates realistic drag and lift forces. For non-spinning objects, the drag force is usually expressed as $F_{D}=\frac{1}{2} C_{d}(\mathrm{Re}) \rho V^{2} S$ where $S$ is the cross sectional area of the Magnus glider (here, $S=2 R L)$ and $C_{d}(\mathrm{Re})$ the drag coefficient, which depend on Re [24]. Here, and following the notations widely used in the literature, the drag and lift forces are expressed as $[3,17,22,23]$ :

$$
\begin{aligned}
& \mathbf{F}_{D}=C_{d}\left(\operatorname{Re}, S_{p}\right) \frac{\rho S V^{2}}{2} \tilde{\mathbf{v}} \\
& \mathbf{F}_{L}=C_{l}\left(\operatorname{Re}, S_{p}\right) \frac{\rho S V^{2}}{2} \tilde{\omega} \times \tilde{\mathbf{v}},
\end{aligned}
$$

where $\tilde{\mathbf{v}}$ is the velocity unit vector and $\tilde{\omega}$ the angular velocity unit vector and where the drag and lift coefficients
$C_{d}$ and $C_{l}$ depend on the Reynolds and Spin numbers. These coefficients have been investigated extensively for cylinders of various aspect ratio, since the seminal work of Magnus on a brass cylinder, followed by successive investigations by Lafay $[27,28]$ and by the group led by Prandtl in Göttingen [29]. The experimental and numerical investigations of the evolution of drag and lift coefficients of spinning cylinders have been compiled by Swanson [22], and more recently by Baladamenti [23]. Most of these studies were led in the context of technical applications such as Flettner boats [19] or other aeronautical ships or airplanes [20] and provide a useful database for the modeling of the aerodynamical forces applied to the Magnus glider. In the next paragraph, we extrapolate the values of the drag and lift coefficients presented in previous studies to the geometry of our Magnus glider.

For non-spinning objects, and in the turbulent regime (i.e. Reynolds numbers in excess of $10^{3}$ ), it is well-known that the drag coefficient is Re-independent [24]. A similar turbulent regime was also observed for spinning objects $[22,23]$; we will thus consider drag and lift coefficients to be Re-independent (below the drag crisis occurring around $R e \sim 4 \times 10^{5}$ for cylinders, an order of magnitude larger than the Reynolds numbers reached in this study). The values of the drag and lift coefficients for cylinders strongly depend on various control parameters [23] such as the the cylinder aspect ratio, the roughness of the surface, the presence of endcaps, etc. Among the measurements of the drag and lift coefficients in wind- 

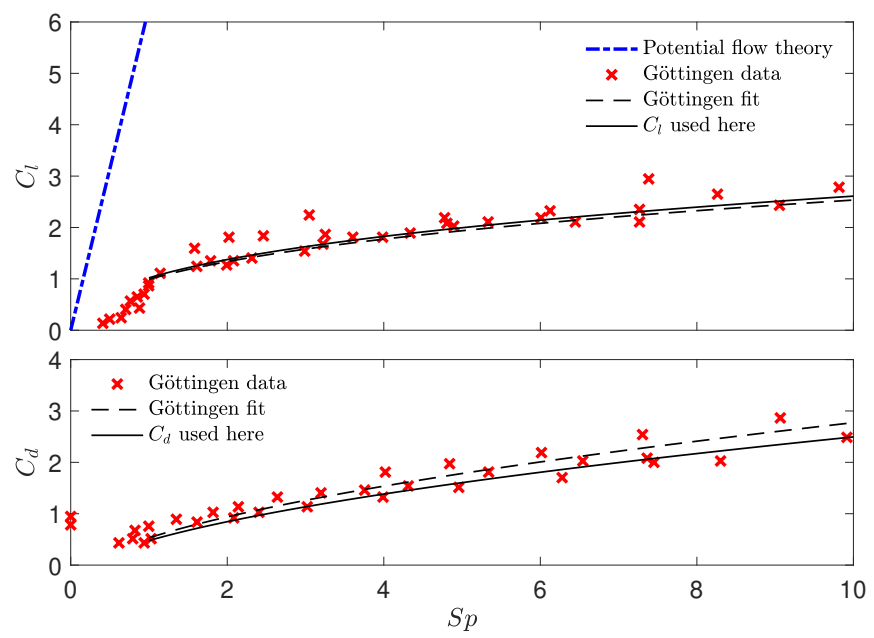

FIG. 4. (Top) Lift and (bottom) drag coefficients as a function of the Spin number $S_{p}$ from the Göttingen wind tunnel (red crosses) and fitted curves for a 1.7 aspect ratio. The blue dash-dotted line corresponds to the potential flow theory.

tunnels for spinning cylinders of various aspect ratios, the closest configuration to our case was studied for an aspect ratio 1.7 [30] in the Göttingen wind tunnel, nearly 90 years ago. We extracted the experimental data from the Göttingen study, shown in Fig. 4 as red crosses. Note that, in the framework of the potential flow theory discussed above, the lift coefficient reads $C_{l}^{p f}=2 \pi S_{p}$ and its evolution is reported as a blue dash-dotted line in Fig. 4. Note that $C_{l} \propto S_{p}$ means that the Magnus force scales as the product of the translational velocity with the rotational velocity. The first important observation is that the lift coefficient extracted from the Göttingen windtunnel experiment is at least one order of magnitude lower than the one computed from potential flow theory. The second observation is that both $C_{l}$ and $C_{d}$ strongly depend on the Spin number $S_{p}$. Fits of the Göttingen experimental data for $S_{p}>1$ (i.e. in the range of Spin numbers reached here) are shown as dashed black lines in Fig. 4, respectively as $C_{d}^{\mathrm{AR}=1.7}=-0.35+0.88 S_{p}^{0.55}$ and $C_{l}^{\mathrm{AR}=1.7}=-0.31+1.3 S_{p}^{0.34}$. We stress that the Göttingen experimental points are somewhat scattered around the fits. The compilation provided by Badalamenti [23] shows that both $C_{l}$ and $C_{d}$ depend on the aspect ratio of the cylinder. We thus choose to model the aerodynamic forces in Eq. 1 using a drag and a lift coefficients proportional to the Göttingen data shown in Fig. 4, i.e equal to $\beta_{d} C_{d}^{\mathrm{AR}=1.7}$, and $\beta_{l} C_{l}^{\mathrm{AR}=1.7}$, with $\beta_{l, d} \sim 1$, since the aspect ratio of the Magnus glider $L /(2 R)$ is equal to 2.67 .

The values of $\beta_{l}$ and $\beta_{d}$ are obtained from the best fits of nine experimental trajectories, reported in Fig. 5 (red solid lines), for various values of the initial kinematic parameters of the Magnus glider. Equation 1 is integrated numerically using as initial conditions the values of $\omega_{0}$, $V_{0}$ and $\alpha_{0}$ obtained experimentally for the nine trajectories [31]. A further approximation is to consider that
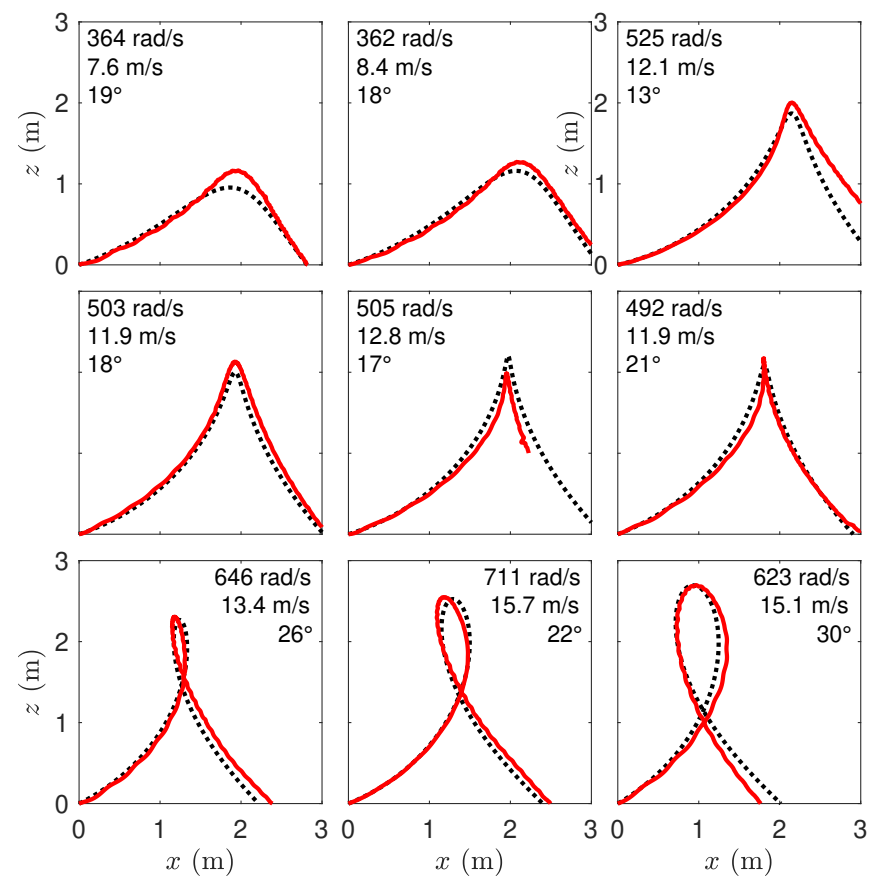

FIG. 5. Nine experimental trajectories (solid red line) and best fit (dashed black line) using the model of Eq. 1, with the drag and lift coefficients shown in Fig. 4. Initial conditions $\omega_{0}$, $V_{0}$ and $\alpha_{0}$ at $(x, z)=(0,0)$, computed from the experimental data, are specified for each trajectory.

the angular velocity is kept constant during the flight of he Magnus glider - this assumption remains valid as long as the flight duration is lower than $\tau_{0}$ and since the drag and lift coefficients only weakly depend on the Spin number for values larger than 2 . The numerically-integrated trajectories are displayed in Fig. 5 (dotted black lines), and correspond to $\beta_{l}=1.03$ and $\beta_{d}=0.9$. The evolution of the drag and lift coefficients used for the numerical integration are shown in Fig. 4 (solid black line) as a function of the Spin number, and are in agreement with the wind-tunnel measurements. The trajectories computed from numerical integration of the model and displayed in Fig. 5 show a very good agreement with the experimental trajectories. As a partial conclusion, we showed here that the modeling of the experimental trajectories is very accurate when using the lift and drag coefficients obtained from wind tunnel measurements [30] for cylinders, in configurations close to our Magnus glider and assuming a constant angular velocity. A possible extension to this work would be a precise experimental characterization of the aerodynamic coefficients of joined truncated cones. We stress here that the remarkable agreement between the experiments and the model is observed although the Magnus glider is a very light projectile whose trajectory may be strongly influenced by external perturbations (weak draft or air convection).

Finally, we note that a further refinement of the model could be incorporated from an added mass effect, since the mass of air $m_{\text {air }}$ in the volume of the Magnus glider 


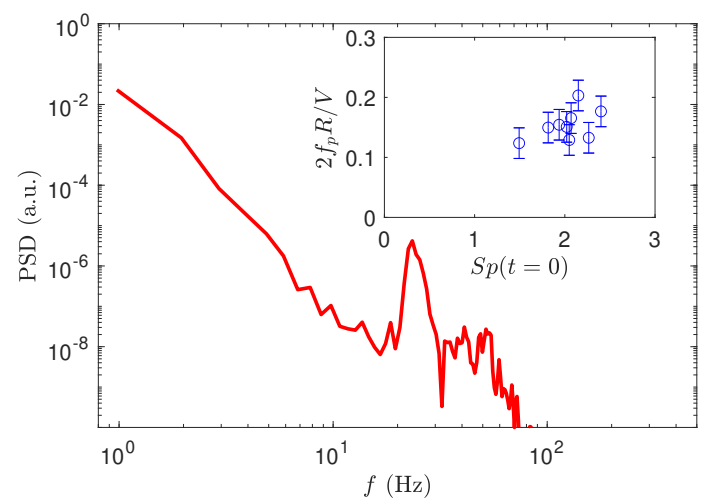

FIG. 6. Power spectral density (PSD) of the horizontal component of position $x(t)$ as a function of frequency $f$ for a typical trajectory, showing a clear peak at $f_{p}$. Inset: evolution of the peak frequency $f_{p}$, expressed as a Strouhal number, as a function if the initial Spin number.

is $4 \%$ of the mass of the Magnus glider. This refinement would lead to consider the following equation of motions: $\left(m+m_{\text {air }}\right) \mathbf{a}=m \mathbf{g}+\mathbf{F}_{L}+\mathbf{F}_{D}$. This refinement only marginally affects the results and does not modifies the message carried here: incorporating the added mass effect leads to $\beta_{l}^{a m}=1.12$ and $\beta_{d}^{a m}=1$.

\section{B. Fast oscillations of the glider}

A closer observation of the trajectories (or the velocities) in Fig. 3 shows strong oscillations around the time-averaged trajectory. The power spectral density of the horizontal component $x(t)$ of a typical trajectory is displayed in Fig. 6, showing a well-defined peaks at $f_{p}=21 \mathrm{~Hz}$ for the fast oscillations of the glider. The origin of these oscillations is now discussed. The inset of Fig. 6 shows the evolution of the peak frequency $f_{p}$, expressed as a Strouhal number $2 f_{p} R / V_{0}$ as a function of the Spin number at time $t=0$ for the nine trajectories reported in Fig. 5. While we cannot rule out that these oscillations are excited by perturbations during the launching phase of the Magnus glider, a probable origin lies in the wake detachment from the Magnus glider at early times. Indeed, the evolution of $2 f_{p} R / V_{0}$ is compatible with the evolution of the Strouhal number as a function of the Spin number, where the Strouhal number is defined as $2 f R / V_{0}$ with $f$ the vortex-shedding frequency. The compilation by Badalamenti [23] indeed shows that the Strouhal number lies between 0.1 and 0.25 and increases with the Spin number: all the features observed here are thus compatible with oscillations excited from vortex-shedding at early times of the trajectory.

\section{Friction decreases angular velocity}

Let us now discuss the time evolution of the angular velocity of the Magnus glider, as reported in the central panel of Fig. 3. From top to bottom, the characteristic times $\tau_{0}$ are respectively $7.1 \mathrm{~s}, 6.1 \mathrm{~s}$, and $4.4 \mathrm{~s}$. The decay of the angular velocity is due to the friction within the boundary layer of the spinning Magnus glider. In wind-tunnel experiments, the amplitude of the dissipation is usually quantified from torque measurements. The torque $T$ required to spin a cylinder at angular velocity $\omega$ in the presence of a cross-flow $V$ is expressed as $T=C_{T} \rho V^{2} R^{2} L$ with a torque coefficient $C_{T}$ and assuming a turbulent friction force, scaling as $\rho V^{2}$, applied uniformly on the surface of the cylinder. The compilation of the estimates of $C_{T}$ from previous studies [23] shows that $C_{T}$ scales linearly with $S_{p}$ as $C_{T} \sim c S_{p}$, with $c$ between 0.02 and 0.06 (this indeed strongly depends on the roughness of the surface). Assuming the moment of inertia of the Magnus glider being that of a cylindrical shell of mass $m$ and radius $R$, Newton's law of rotation reads

$$
m R^{2} \frac{\mathrm{d} \theta^{2}}{\mathrm{~d} t^{2}}=-C_{T}\left(S_{p}\right) \rho V^{2} R^{2} L \sim-c \omega \rho V R^{3} L,
$$

and since $\omega=\mathrm{d} \theta / \mathrm{d} t$,

$$
\frac{\mathrm{d} \omega}{\mathrm{d} t} \sim-\frac{c \omega \rho V R L}{m} .
$$

The angular velocity thus decays exponentially with a characteristic time $\frac{m}{c \rho V R L}$. Assuming $c=0.05$ (in the range from the literature), the estimates for the decay times are respectively $8.1,5.8$ and $4.5 \mathrm{~s}$ for $V_{0}$ equal $8.4,11.9$ and $15.1 \mathrm{~m} / \mathrm{s}$ respectively, which is in excellent agreement with the experimental observations.

\section{WHEN DOES THE MAGNUS GLIDER LOOP BACK ?}

In the previous section, we introduced a model for the drag and lift forces that accurately describe the aerodynamic forces applied to the Magnus glider. In this section, we solve numerically the equations of motion to identify which of the three types of trajectories are observed as a function of the initial kinematic parameters $V_{0}, \alpha_{0}$ and $\omega_{0}$. Indeed, due to our experimental protocol using a single rubber band to launch and spin the Magnus glider, there is a strong correlation between the amplitude of the initial velocity $V_{0}$ and the initial angular velocity $\omega_{0}$. More specifically, for three values of $\omega_{0}$ (respectively to 360,500 and $660 \mathrm{rad} / \mathrm{s}$ ), Eq. 1 is solved numerically for various values of $V_{0}$ and $\alpha_{0}$, using the values of the drag and lift coefficients introduced in the previous section. The angular velocity was assumed to remain constant. The results are reported in Fig. 7, 

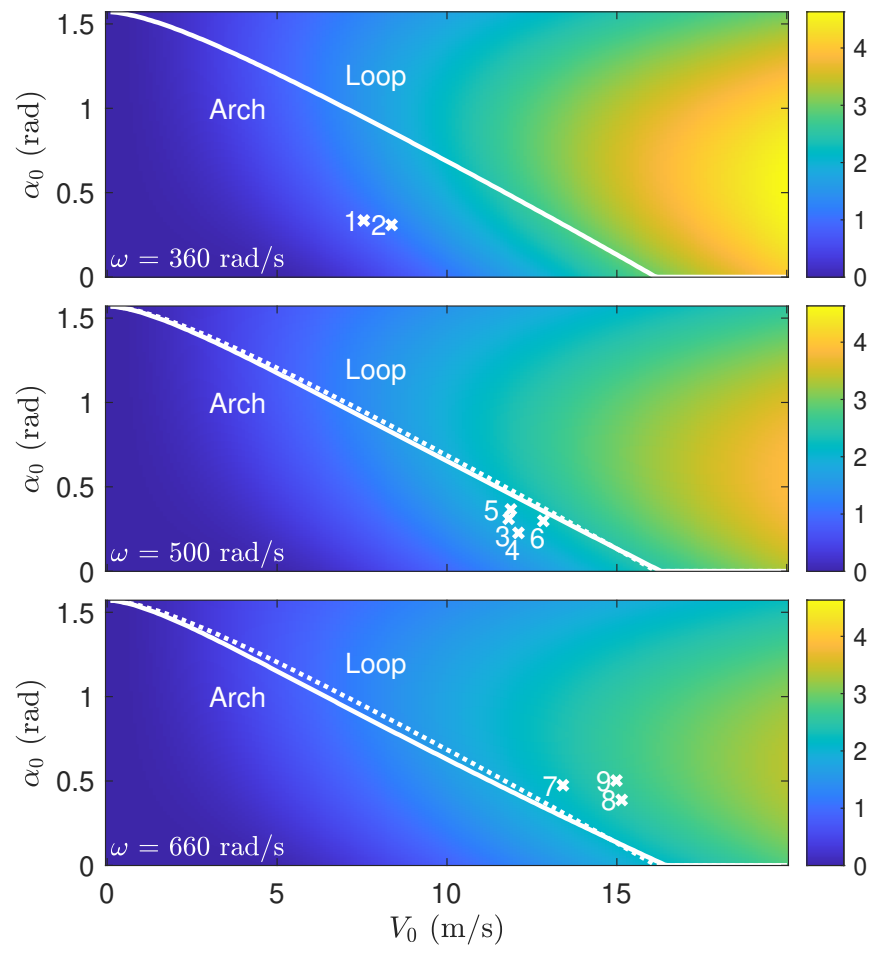

FIG. 7. Phase diagram of behavioral regimes, color-coding of the altitude of the apex of the trajectory (in meters) in the parameter space $\left(V_{0}, \alpha_{0}\right)$ for $\omega_{0}$ equal to (top) $360 \mathrm{rad} / \mathrm{s}$, (middle) $500 \mathrm{rad} / \mathrm{s}$ and (bottom) $650 \mathrm{rad} / \mathrm{s}$. The white lines display the location of the cusps. See text for details.

where the steps are $0.1^{\circ}$ for $\alpha_{0}$ and $0.1 \mathrm{~m} . \mathrm{s}^{-1}$ for $V_{0}$. The white lines correspond to trajectories where the horizontal velocity vanishes, i.e. at the apex of the cusps: this line is thus a separatrix between arches, on the left, and loops, on the right. The white dashed line in the middle and bottom panels corresponds to the separatrix shown as a solid line in the top panel. As a first conclusion, the angular velocity has a weak effect on the type of trajectory. A second rather counter-intuitive conclusion can be drawn from the inspection of the colorbar of Fig. 7 which represents the altitude of the apex of the trajectory (in meters): higher altitudes are reached at lower angular velocities. These results are easily explained from the evolution of the aerodynamic coefficients with the Spin number as shown in Fig. 4 for Spin numbers larger than 2. Indeed, the drag coefficient increases faster than the lift coefficient with the Spin number. Thus, a higher angular velocity leads to a higher drag, and thus lower altitude, while it does not strongly modifies the lift force. The white crosses labeled 1 to 9 in Fig. 7 correspond to the values of $V_{0}$ and $\alpha_{0}$ for the trajectories reported Fig. 5 (1 referring to the top-left trajectory and 9 to the bottom right trajectory). Trajectories 1 to 4 indeed correspond to arches. Trajectories 5 and 6 lie very close to the white line, i.e. correspond to cusps, while trajectories 7 to 9 are looped trajectories. As a partial conclusion here, we demonstrated that the type of trajectory only weakly de- pends on $\omega_{0}$ and mainly depends on the amplitude and orientation of the initial velocity $\mathbf{V}_{\mathbf{0}}$, while the height of the apex strongly depends on $\omega_{0}$.

\section{CONCLUSION}

In this article we described an experimental investigation of the dynamics of Magnus gliders made from light paper cups. Such gliders are a simple, inexpensive and easy to image in a classroom laboratory allowing to measure the spectacular effect of the Magnus lift on the trajectories of spinning objects. The analysis of high-speed movies revealed three types of trajectories: arches, cusps and loops. These trajectories were accurately modeled using simple analytical descriptions for the drag and lift forces, based on previous aerodynamical studies in wind tunnels. The model predicts that the type of trajectory mainly depends on the amplitude and orientation of the launching velocity $\mathbf{V}_{\mathbf{0}}$. The altitude of the apex strongly depends on the spin for a given launching velocity, and, counter-intuitively, decreases with increasing spin. These features are readily understood from the evolution of the drag and lift forces with the Spin number.

The investigation of the dynamics of Magnus gliders in the vicinity of the drag crisis, i.e. for Reynolds numbers around $410^{5}$, where the drag force suddenly decreases would undoubtedly be a natural follow-up of our study. The influence of the reverse Magnus effect [17], linked to the difference in the boundary layers on the upstream and downstream sides could also lead to unexpected spectacular trajectories. We addressed the aerodynamic origin of small amplitudes fast oscillations on the trajectories of a symmetric Magnus glider, but similar oscillations could also be triggered by asymmetric gliders i.e., those for which the center of mass is off the axis of rotation.

\section{ACKNOWLEDGMENTS}

The authors acknowledge support from the University of Lyon, the Université Claude Bernard Lyon 1, and the École Normale Supérieure de Lyon. The authors are thankful the committees of the French Physicists' Tournament and of the International Physicists' Tournament. The authors are grateful to L. Delance, C. Desgrange, R. Faure, L. Guislain, L. Morlet-Decarnin, H. Mussillon, A. Walbecq and P. Wang for fruitful discussions.

\section{Appendix A: Building and launching Magnus gliders: technical details and dead ends}

The Magnus gliders used for this study were made out of paper cups, of height $L / 2=9 \mathrm{~cm}$, bottom radius 2.75 $\mathrm{cm}$, and top radius $4 \mathrm{~cm}$, glued together at their bottoms. They were launched using long rubber bands assembled 
from domestic rubber elastic bands. The domestic rubber bands were $1 \mathrm{~cm}$ wide, $1 \mathrm{~mm}$ thick and $10 \mathrm{~cm}$ long and several of them (between 5 and 10 depending on the experiment) were connected using a loop to loop knots. The resulting band was wrapped around the center of the Magnus glider (i.e. taking advantage of the reinforced rim of the bottoms of the cups) with constant tension provided by a weight (between $1 \mathrm{~kg}$ and $3 \mathrm{~g}$, depending on the experiment) attached at the bottom the band, and hanging 1 or $2 \mathrm{~m}$ below the Magnus glider. The free end of the rubber was then firmly held while the other end was stretched at a length $l$ between 50 and $80 \mathrm{~cm}$ (i.e. roughly twice the length of the un-stretched remaining part of the band).

Several type of cups were tested, motivated by the variety of Magnus gliders reported in the video by Bruce Yeany [18]. Styrofoam cups are extremely light, but also extremely brittle, which restrict the ranges of tension and stretching of the rubber without breaking the Magnus gliders. Light plastic cups were also too fragile to sustain the wrapping the rubber band. We also tested small paper cups and paper towel rolls, but their trajectories were very unstable (i.e. the trajectories did not remain $2 \mathrm{D}$ ).
The reinforced bottom rims of paper cups were found to be an asset for the mechanical strength of the assembly.

\section{Appendix B: High speed imaging requirements}

High speed imaging of the trajectories require a resolution larger than 1200 by 700 pixels. The capture of the rotational dynamics (around $100 \mathrm{rev} / \mathrm{s}$ ) requires a framerate larger than a few hundreds of frames per seconds. In our manuscript we indeed use an ultra high speed camera, but at a moderate frame-rate of $4000 \mathrm{fps}$. We stress here that many of the cameras available in undergrad labs would allow to correctly image the experiments. Using standard mid-range smartphones which routinely image up to $240 \mathrm{fps}$ in 2021 would be sufficient to image the trajectories (with a far better resolution than what we report in this study). The rotation rate for all of the regimes reported in our manuscript could be imaged using high-end smartphones which image up to $1920 \mathrm{fps}$ in 2021 with a $1280 \times 720$ pixels resolution.
[1] G. Magnus, On the Deflection of a Projectile, Poggendorf's Annalen der Physik und Chemie 88, 604 (1853).

[2] W. Johnson, The magnus effect - early investigations and a question of priority, International Journal of Mechanical Sciences 28, 859 (1986).

[3] C. Clanet, Sports ballistics, Annual Review of Fluid Mechanics 47, 455 (2015).

[4] C. Frohlich, Resource letter ps-2: Physics of sports, American Journal of Physics 79, 565 (2011).

[5] J. E. Goff, J. Kelley, C. M. Hobson, K. Seo, T. Asai, and S. B. Choppin, Creating drag and lift curves from soccer trajectories, European Journal of Physics 38, 044003 (2017).

[6] J. E. Goff and M. J. Carré, Trajectory analysis of a soccer ball, American Journal of Physics 77, 1020 (2009).

[7] A. M. Nathan, The effect of spin on the flight of a baseball, American Journal of Physics 76, 119 (2008).

[8] J. Thomson, The dynamics of a golf ball, Nature 85, 251-257 (1910).

[9] A. R. Penner, The physics of golf, Reports on Progress in Physics 66, 131 (2002).

[10] A. Štěpánek, The aerodynamics of tennis balls - the topspin lob, American Journal of Physics 56, 138 (1988).

[11] M. Peastrel, R. Lynch, and A. Armenti, Terminal velocity of a shuttlecock in vertical fall, American Journal of Physics 48, 511 (1980).

[12] C. Cohen, B. D. Texier, D. Quéré, and C. Clanet, The physics of badminton, New Journal of Physics 17, 063001 (2015).

[13] R. D. Lorenz, Spinning Flight (Springer, New York, NY, 2006).

[14] G. Dupeux, C. Cohen, A. L. Goff], D. Quéré, and C. Clanet, Football curves, Journal of Fluids and Structures 27, 659 (2011).
[15] M. McBeath, A. Nathan, A. Bahill, and D. Baldwin, Paradoxical pop-ups: Why are they difficult to catch?, American Journal of Physics 76, 723 (2008).

[16] A. M. Nathan, Analysis of knuckleball trajectories, Procedia Engineering 34, 116 (2012).

[17] J. Bush, The aerodynamics of the beautiful game, In Sports Physics , 171 (2013).

[18] B. Yeany, Physics of toys- cup flyers (2015), https:// www youtube. com/watch?v=05zF0sBwHe8.

[19] A. Flettner, The Flettner Rotorship, Journal of Engineering 19, 117-120 (1925).

[20] J. Seifert, A review of the magnus effect in aeronautics, Progress in Aerospace Sciences 55, 17 (2012).

[21] This is achieved using basic image processing from Matlab software, including imfindcircles, whose output are the centers and radii of the disks detected in each image.

[22] W. M. Swanson, The Magnus Effect: A Summary of Investigations to Date, Journal of Basic Engineering 83, 461 (1961).

[23] C. Badalamenti, On the application of rotating cylinders to micro air vehicles (2010).

[24] D. J. Tritton, Physical Fluid Dynamics (Springer, Dordrecht, 1977).

[25] F. F. Chen, Introduction to Plasma Physics and Controlled Fusion (Springer, Cham, 2016).

[26] P. M. Bellan, Fundamentals of Plasma Physics (Cambridge University Press, 2006).

[27] M. A. Lafay, Sur l'inversion du phénomène de magnus, comptes-rendus, Académie des Sciences 151, 867 (1910).

[28] M. A. Lafay, Contribution Expérimentale à l'Aérodynamique du Cylindre (H. Dunod et E. Pinat, 1912).

[29] L. Prandtl, Application of the magnus effect to the wind propulsion of ships, Technical Report TM-367 37 (1926). 
[30] A. Busemann, Messungen an Rotierenden Zylindern, In Ergebnisse der Aerodynamik Versuchsanstalt zu
Göttingen IV, 101 (1931).

[31] Using the ode45 Matlab function. 